\title{
Identification of foods that affect the anti-cancer activity of pitavastatin in cells
}

\author{
MOHAMMED J. JAWAD, SUAD IBRAHIM, MAYUR KUMAR, CHARLIE BURGERT, \\ WEN-WU LI and ALAN RICHARDSON
}

The Guy Hilton Research Centre, School of Pharmacy and Bioengineering, Keele University, Stoke-on-Trent, Staffordshire ST4 7QB, UK

Received September 19, 2021; Accepted November 19, 2021

DOI: $10.3892 / 01.2022 .13193$

\begin{abstract}
Statins inhibit the synthesis of mevalonate, a precursor isoprenoid molecule to geranylgeraniol that is necessary for the post-translational modification of several small GTPase oncogenes. Despite numerous preclinical studies suggesting that statins can be effective anticancer agents, prospective clinical trials have failed to demonstrate any clinical benefit in patients with cancer. We previously demonstrated that geranylgeraniol suppresses the activity of statins in cell culture studies, and that pitavastatin can cause regression of ovarian cancer xenografts in mice if the animals' diet is modified to avoid the inclusion of geranylgeraniol. Dietary sources of geranylgeraniol may consequently limit the activity of statins in cancer clinical trials. The present study tested several foods to identify those that affected the cytotoxic activity of pitavastatin towards ovarian cancer cells. Solvent extracts of several foods were tested for their ability to suppress the effects of pitavastatin in cell growth assays. The results revealed that pitavastatin induced cell death in ovarian cancer cells $\left(\mathrm{IC}_{50}=5.2 \mu \mathrm{M}\right)$ and this was blocked by geranylgeraniol whereas other products of the mevalonate pathway (coenzyme Q, dolichol or cholesterol) had no effect on the activity of pitavastatin in cell growth assays. Solvent extracts from several foods, especially oils (apart from rapeseed), also blocked the cytotoxic activity of pitavastatin. Several extracts from a range of fruit, vegetables and carbohydrate-rich foods also did not block the activity of pitavastatin. However, extracts from beans, lettuce, oats, eggs and various nuts reduced the activity of pitavastatin. These data identified foods that patients could eat to potentially improve the outcome of clinical trials of pitavastatin in cancer.
\end{abstract}

Correspondence to: $\mathrm{Dr}$ Alan Richardson, The Guy Hilton Research Centre, School of Pharmacy and Bioengineering, Keele University, Thornburrow Drive, Hartshill, Stoke-on-Trent, Staffordshire ST4 7QB, UK

E-mail: a.richardson1@keele.ac.uk

Key words: statin, ovarian cancer, pitavastatin, food, geranylgeraniol

\section{Introduction}

Ovarian cancer is the second most common gynaecological malignancy following uterine corpus cancer and it is considered the fifth leading cause of cancer death in women with more than 4,000 deaths annually in UK (1). In addition, it has been called a 'silent killer' because no specific symptoms have been associated with the early stages of the disease. The 5-year survival rate for patients diagnosed with stage II or greater ovarian cancer is $\sim 40 \%$. Although there have been recent improvements in treatment, notably the introduction of PARP inhibitors, it still represents a significant unmet medical problem and new treatments are required.

Statins are a class of low molecular weight drugs that are used effectively to control hypercholesterolemia. They inhibit hydroxymethylglutaryl Coenzyme-A reductase (HMGCR). Statins reduce production of mevalonate, a precursor in the synthesis of cholesterol and isoprenoids such as farnesol and geranylgeraniol (2). A significant body of evidence suggests that statins may be repurposed to treat cancer (3). Numerous studies have reported that statins can induce cancer cell death through apoptosis [reviewed in (3)] as a result of reducing the production of geranylgeraniol. This isoprenoid is necessary for the proper function of small GTPase oncogenes which are consequently inactivated by statins (4). Retrospective analyses have shown reduced cancer mortality in patients using statins to control elevated cholesterol (3). However, prospective clinical trials of statins in cancer patients have so far failed to show any survival benefit. We have argued that this is due in part to failures in trial designs which have not adequately considered pharmacodynamic and pharmacokinetic factors (5).

In addition to these factors, diet may affect the efficacy of statins in treating cancer (6). We showed that pitavastatin can cause the regression of ovarian cancer xenografts in mice. However, this required the use of a diet that lacked geranylgeraniol. Supplementation of the diet with geranylgeraniol restored the growth of the xenografts in mice receiving pitavastatin (6). Although others have also reported that statins can affect the growth of cancer xenografts, to our knowledge no other researchers have observed tumour regression, nor have they controlled dietary geranylgeraniol. Considering that several human foods, particularly oils (7), have been shown to contain 
geranylgeraniol, this raises the concern that dietary geranylgeraniol could also interfere with the anti-cancer activity of statins in patients and that clinical trials of statins may not be successful unless diet is adequately controlled. Prospective clinical trials of statins to treat cancer to date have uniformly failed to consider the effect of dietary geranylgeraniol in their design (5). Although there are geranylgeraniol-free complete food products (e.g., Ensure) that could be used by patients on a statin clinical trial, these liquid foods may not be suitable for prolonged use due to poor patient compliance with the liquid diet. Thus, it is desirable to identify additional foods for trial participants that are less likely to interfere with the activity of pitavastatin.

We have tested several foods to identify those which interfere with the cytotoxic activity of statins. We have made use of the observation that the addition of geranylgeraniol, or the organic extract of some foods, can suppress the cytotoxic activity of statins $(6,8)$ against ovarian cancer cells. Here we use that bioassay to identify several foods which do not interfere with the activity of pitavastatin and may potentially be eaten by patients during a clinical trial. Importantly we also identify foods which suppress the activity of pitavastatin and so should be avoided during clinical trials of statins.

\section{Materials and methods}

Materials. Thirty foodstuffs were obtained for the purposes of extracting fats and were obtained from different local markets in the United Kingdom. Pitavastatin (Sequoia Research Products), geranylgeranyl (Sigma-Aldrich), mevalonate (Enzo Life Sciences), isopentenol (Sigma-Aldrich), dolichol (Avanti) and Coenzyme Q10 were dissolved in DMSO (20 mM). Cell culture media were obtained from Lonza.

Preparation of extracts. Extracts of the different foods were prepared according to the method reported by Muraguchi (9). Each solid foodstuff $(50 \mathrm{~g})$ was homogenized in an electric blender and then transfer to a mortar and homogenised manually with a pestle in $60 \mathrm{ml}$ methanol. Then, $30 \mathrm{ml}$ of chloroform/methanol (50/50\%) were added and the extract homogenized briefly again. The samples were filtered through fluted filter paper. Oils $(50 \mathrm{~g})$ were directly mixed with methanol and chloroform/methanol without homogenization. Both types of extracts were transferred to a separating funnel and the upper layer collected and evaporated. The dried residues were dissolved in $25 \mathrm{ml} 99 \%$ ethanol and $25 \mathrm{ml} 5 \mathrm{M}$ potassium hydroxide were added and the solution incubated at $56^{\circ} \mathrm{C}$ for $1 \mathrm{~h}$ in a water bath. After cooling and neutralisation with $25 \mathrm{ml} 5 \mathrm{M}$ hydrochloric acid and addition of $30 \mathrm{ml}$ water, the resulting solution was partitioned with $120 \mathrm{ml}$ n-hexane. The upper phase was collected and the solvent removed in a rotary evaporator and then in a freeze dryer overnight. The residue was dissolved in $1 \mathrm{ml} \mathrm{DMSO}$ and stored at $-20^{\circ} \mathrm{C}$ for later analysis.

Cell growth assays. Ovcar-4 and Fuov-1 cells were grown in RPMI 1640 medium supplemented with $10 \%$ FCS, $2 \mathrm{mM}$ glutamine and $50 \mu \mathrm{g} / \mathrm{ml}$ penicillin/streptomycin. Cells were incubated at $37^{\circ} \mathrm{C}$ and in a humidified $5 \% \mathrm{CO}_{2}$ atmosphere.
Cells were regularly tested to confirm absence of mycoplasma infection.

Cells $(5,000 /$ well $)$ were seeded in 96-well plates in $80 \mu \mathrm{l}$ of growth medium. After incubation for $24 \mathrm{~h}$, pitavastatin, geranylgeraniol, or food extracts at the indicated final concentration, either alone or in combination, were added to cells. The cells were incubated for a further $72 \mathrm{~h}$, the growth medium removed and the cells in each well were fixed in $100 \mu 1$ cold $10 \%$ trichloroacetic acid (TCA) for $30 \mathrm{~min}$ on ice. The TCA was removed and the cells were left to air dry, before staining in $0.4 \%$ sulforhodamine B in $1 \%$ acetic acid for $30 \mathrm{~min}$. Excess SRB was removed by washing the wells three times in $1 \%$ acetic acid and the plates were left to dry. Lastly, the dye was solubilised in $100 \mu 110 \mathrm{mM}$ Tris (pH 10) and the absorbance at $570 \mathrm{~nm}$ (A570) was determined using a BioTek Synergy 2 multi-mode microplate reader.

A parameter 'rescue' was defined to quantify the activity of the extracts in suppressing the activity of pitavastatin. Rescue $(\%)=\left[\left(\mathrm{N}_{\mathrm{pe}}-\mathrm{N}_{\mathrm{p}}\right) /\left(\mathrm{N}_{0}-\mathrm{N}_{\mathrm{p}}\right)\right] \times\left(\mathrm{N}_{0} / \mathrm{N}_{\mathrm{e}}\right) \times 100$ in which the relative biomass (used as a surrogate for cell number) measured by SRB staining were measured in samples exposed to either pitavastatin and the extract $\left(N_{p e}\right)$, or pitavastatin alone $\left(N_{p}\right)$, or DMSO (solvent control, $N_{o}$ ) or the extract alone $\left(N_{\mathrm{e}}\right)$. The second term in the equation was included to control for any effect of the extract itself on cell growth. However, if the extract on its own was apparently cytotoxic (here defined as inhibition of growth by more than $15 \%$, i.e., $\left.N_{e} / N_{0}<0.85\right)$ the data were considered unreliable and rejected for further analysis.

Cell viability assays. To estimate the cell viability by trypan blue staining, $2 \mathrm{ml}$ of Ovcar- 4 cells $\left(1 \times 10^{5}\right.$ per $\left.\mathrm{ml}\right)$ were seeded per well of a 6-well plate. After $24 \mathrm{~h}, 20 \mu \mathrm{l}$ of medium containing pitavastatin, geranylgeraniol or extract were added. After $72 \mathrm{~h}$, the medium (containing any detached cells) was collected and combined with the adherent cells which had been detached by trypsinization. The combined samples were centrifuged (150 g, $3 \mathrm{~min})$, the pellet resuspended in $0.5 \mathrm{ml}$ of medium and mixed with an equal volume of $0.4 \%(\mathrm{v} / \mathrm{v})$ trypan blue (Sigma-Aldrich). The cells were counted using a Neubauer haemocytometer.

GC-MS analysis. 1-2 mg of extracts were dissolved in $200 \mu \mathrm{l}$ ethylacetate and sonicated for $5 \mathrm{~min}$ at $40^{\circ} \mathrm{C}$. Subsequently, 1-2 $\mu \mathrm{l}$ of the solution was injected into the gas chromatography mass spectrometer (GC-MS), an Agilent 7890 coupled with Agilent MS type 5975 C MSD (Agilent Technologies). The gas chromatography was started for two minutes with an initial oven temperature of $60^{\circ} \mathrm{C}$ and increased to $300^{\circ} \mathrm{C}$ at a rate of $10^{\circ} \mathrm{C} / \mathrm{min}$, followed by $4 \mathrm{~min}$ at $300^{\circ} \mathrm{C}$ to produce a total run of $30 \mathrm{~min}$ at a steady helium pressure (10 psi). Mass spectral data were acquired in scanning mode within the $40-1,000 \mathrm{~m} / \mathrm{z}$ range.

Statistical analysis. The data obtained from cell growth assays was analysed by using the GraphPad Prism software (GraphPad Software, Inc.). Non-linear regression was used to fit a four-parameter (Hill-equation) sigmoidal dose-response curve to determine $\mathrm{IC}_{50}$ values. Statistical significance was assessed using paired or one sample t-tests where indicated. 
Table I. Mass of extract recovered from $50 \mathrm{~g}$ of foodstuff.

Food

Extract mass, mg

Grape seed oil

580

Corn oil

370

Ground nut oil

350

Rape seed oil

400

Coconut oil

110

Sesame oil

160

Sunflower oil

510

Butter

180

Oats

60

Bread

Pasta

30

Boiled potato

10

Kiwi 10

Lettuce 550

Passion fruit 40

Pomegranate

Cherry

Fig

Squash

Gooseberry

Pears

Tomato

Pecan nuts

Pasta sauce

Cheese

Milk

Strawberry jam

Boiled egg
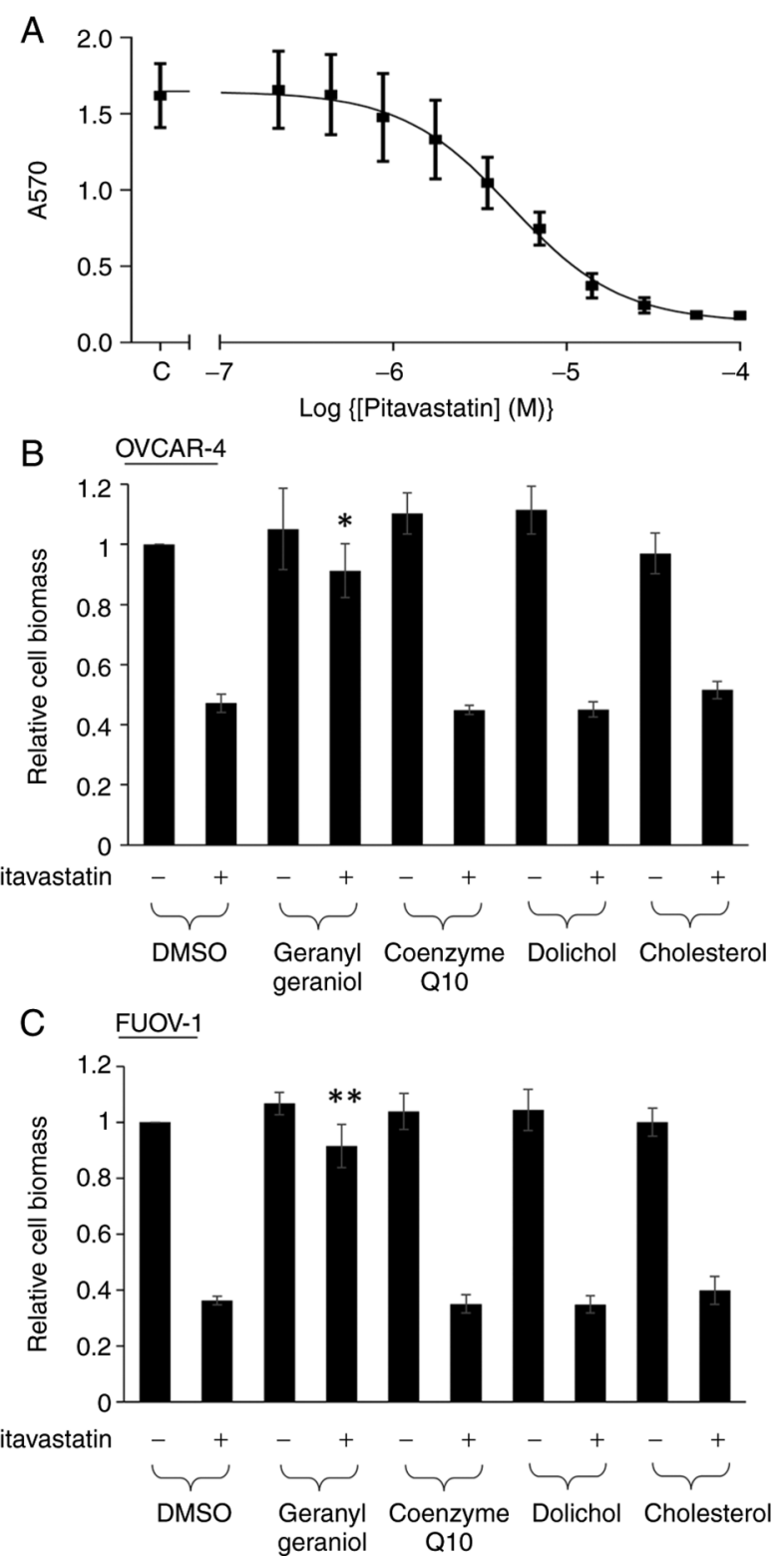

Figure 1. Activity of pitavastatin is reversed by geranylgeraniol but no other mevalonate pathway metabolites. Cells were exposed to pitavastatin $(10 \mu \mathrm{M})$ and/or the indicated metabolites [geranylgeraniol $(5 \mu \mathrm{M})$, coenzyme Q10 $(5 \mu \mathrm{M})$, dolichol $(5 \mu \mathrm{M})$ or cholesterol $(50 \mu \mathrm{M})]$. After $72 \mathrm{~h}$, cell biomass was assessed by staining with sulforhodamine B. (A) Activity of pitavastatin was assessed in Ovcar-4 cell growth assays. Effect of metabolites of the mevalonate pathway on pitavastatin activity against (B) Ovcar-4 or (C) Fuov-1 ovarian cancer cells. The results (mean $\pm \mathrm{SD} ; \mathrm{n}=3$ ) are expressed as a fraction of the absorbance measured in untreated cells. ${ }^{*} \mathrm{P}<0.05$ and ${ }^{* *} \mathrm{P} \leq 0.01$ vs. pitavastatin alone; paired Student's t-test.

completely restores the growth of the cells in the presence of pitavastatin to control levels and $0 \%$ represents an extract that has no effect on growth of the cells in the presence of pitavastatin. By monitoring the effect of the extracts in the absence of pitavastatin, we were also able to determine if any extracts were cytotoxic or promoted cell growth.

The extracts from several oils were able to inhibit the activity of pitavastatin, in particular corn oil, sunflower oil (as previously reported) (6) and grape seed oil (Fig. 2A). However, rape seed oil had minimal activity. We next tested extracts from several fruit and vegetables (Fig. 2B). A number pitavastatin. To quantify this, we defined a parameter 'rescue' (see methods) where a value of $100 \%$ reflects an extract that 

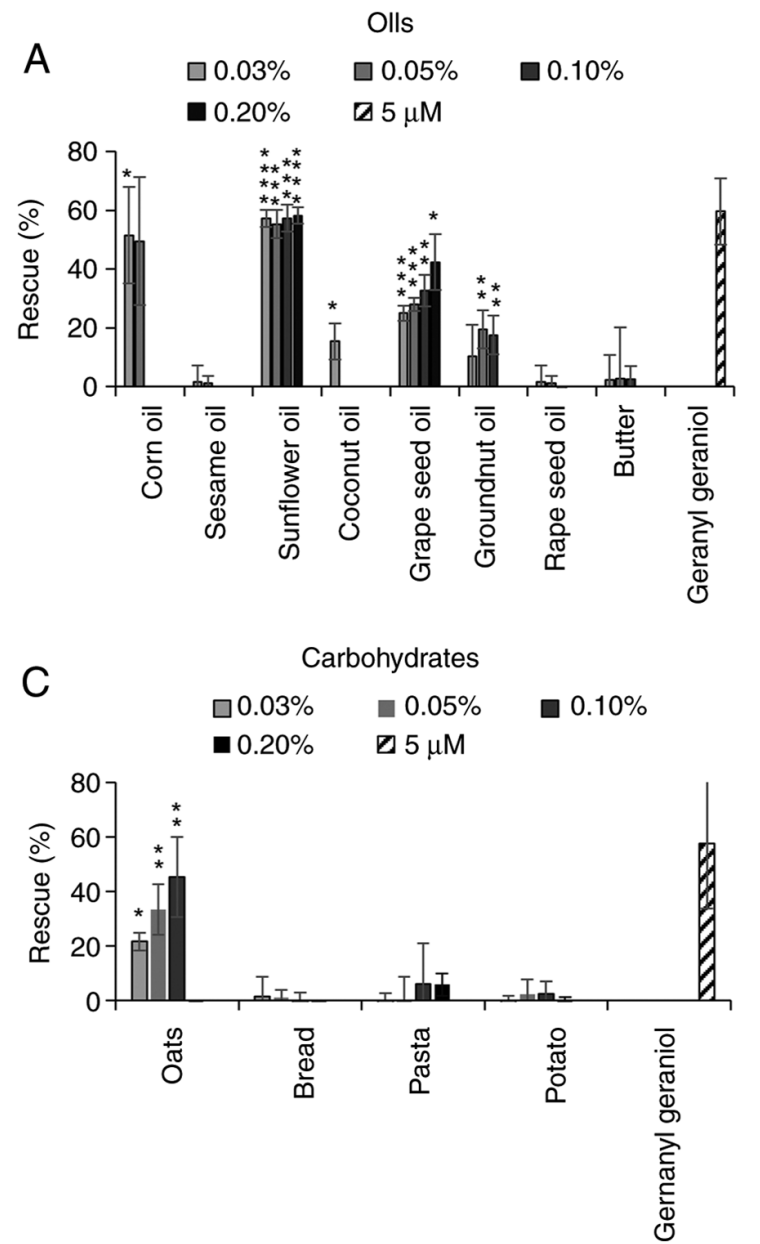
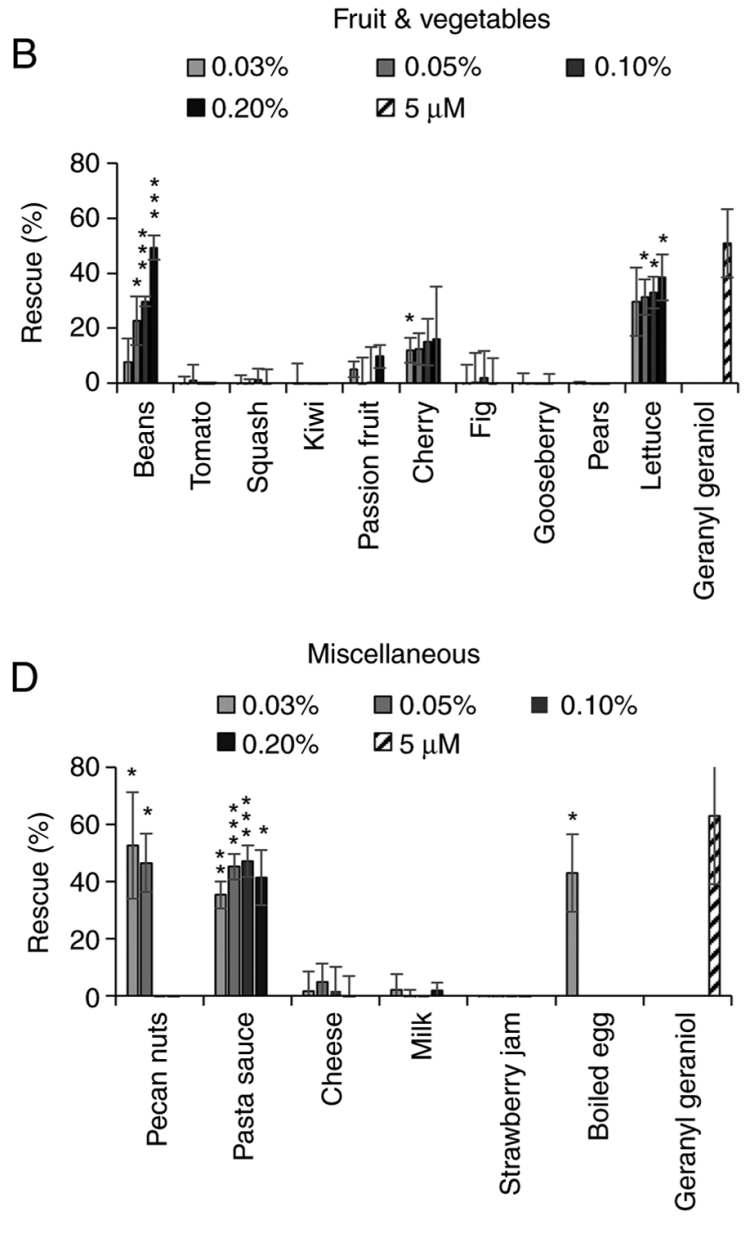

Figure 2. Effect of food extracts on the activity of pitavastatin in Ovcar-4 cells. Effects of extracts from (A) oils, (B) fruit and vegetables, (C) carbohydrates and (D) miscellaneous other foods on the cytotoxic activity of pitavastatin were assessed in Ovcar- 4 cells. The cells were exposed to pitavastatin (10 $\mu \mathrm{M})$ and/or the indicated concentration of food extract. After $72 \mathrm{~h}$, the cell biomass was assessed by staining with sulforhodamine B. The results (mean \pm SD; $\mathrm{n}=3$ ) are expressed as the rescue of the cytotoxic effects of pitavastatin, as defined in the methods section. Geranylgeraniol $(5 \mu \mathrm{M})$ was included as a positive control. Data from corn oil $(0.1$ and $0.2 \%)$, coconut oil $(0.1$ and $0.2 \%)$, groundnut oil $(0.2 \%)$, rape seed oil $(0.2 \%)$, sesame oil $(0.1$ and $0.2 \%)$, pecan nuts $(0.1$ and $0.2 \%)$, oats $(0.2 \%)$ and eggs $(0.05,0.1$ and $0.2 \%)$ have been omitted because these extracts were toxic $\left(>15 \%\right.$ inhibition of cell growth). ${ }^{*} \mathrm{P}<0.05,{ }^{* * *} \mathrm{P}<0.01,{ }^{* * * *} \mathrm{P}<0.005$ and ${ }^{* * * *} \mathrm{P}<0.001$ vs. no rescue; one sample t-test.

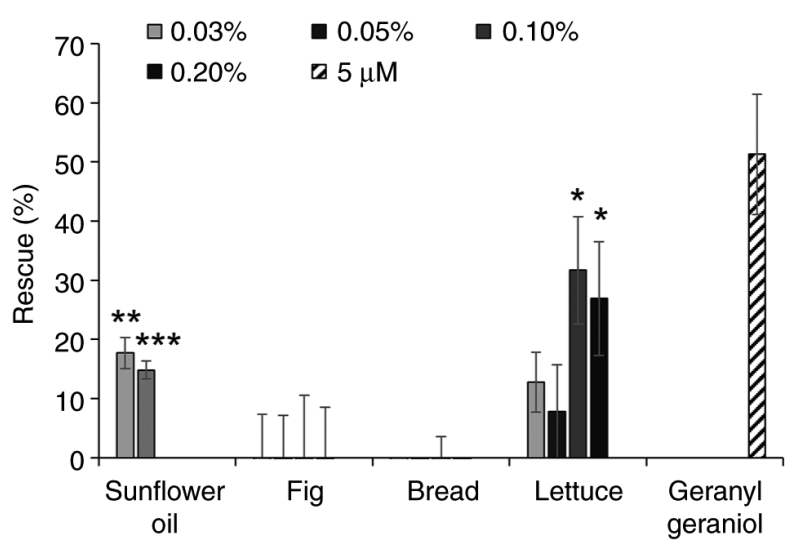

Figure 3. Effect of food extracts on the activity of pitavastatin in Fuov-1 cells. Effect of extracts from various foodstuffs on the cytotoxic activity of pitavastatin was assessed in Ovcar-4 cells. The cells were exposed to pitavastatin $(10 \mu \mathrm{M})$ and/or the indicated food extract and after $72 \mathrm{~h}$, cell biomass was assessed by staining with sulforhodamine $\mathrm{B}$. The results (mean $\pm \mathrm{SD} ; \mathrm{n}=3$ ) are expressed as the rescue of the cytotoxic effects of pitavastatin, as defined in the methods section. Geranylgeraniol $(5 \mu \mathrm{M})$ was included as a positive control. Data from sunflower oil ( 0.1 and $0.2 \%)$ have been omitted because these extracts were toxic ( $>15 \%$ inhibition of cell growth). ${ }^{*} \mathrm{P}<0.05 ;{ }^{* *} \mathrm{P}<0.01$; ${ }^{* * * *} \mathrm{P}<0.005$ vs. no rescue; one sample t-test.

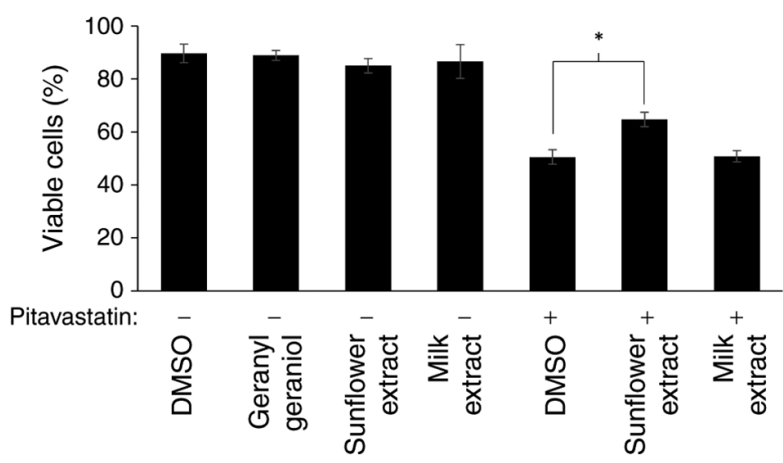

Figure 4. Effect of cell extracts on pitavastatin induced cell death. Ovcar-4 cells were treated with the indicated combinations of pitavastatin $(10 \mu \mathrm{M})$, geranylgeraniol $(10 \mu \mathrm{M})$, sunflower or milk extracts. After $25 \mathrm{~h}$, cell viability was assessed using trypan blue staining. The results (mean $\pm S D ; n=3$ ) were expressed as a percentage of the total number of cells counted in each sample. ${ }^{*} \mathrm{P}<0.005$; paired Student's t-test.

of these did not have any effect on the activity of pitavastatin, however the extracts from beans and cherries were partially able to restore the growth of cells in the presence of pitavastatin. 

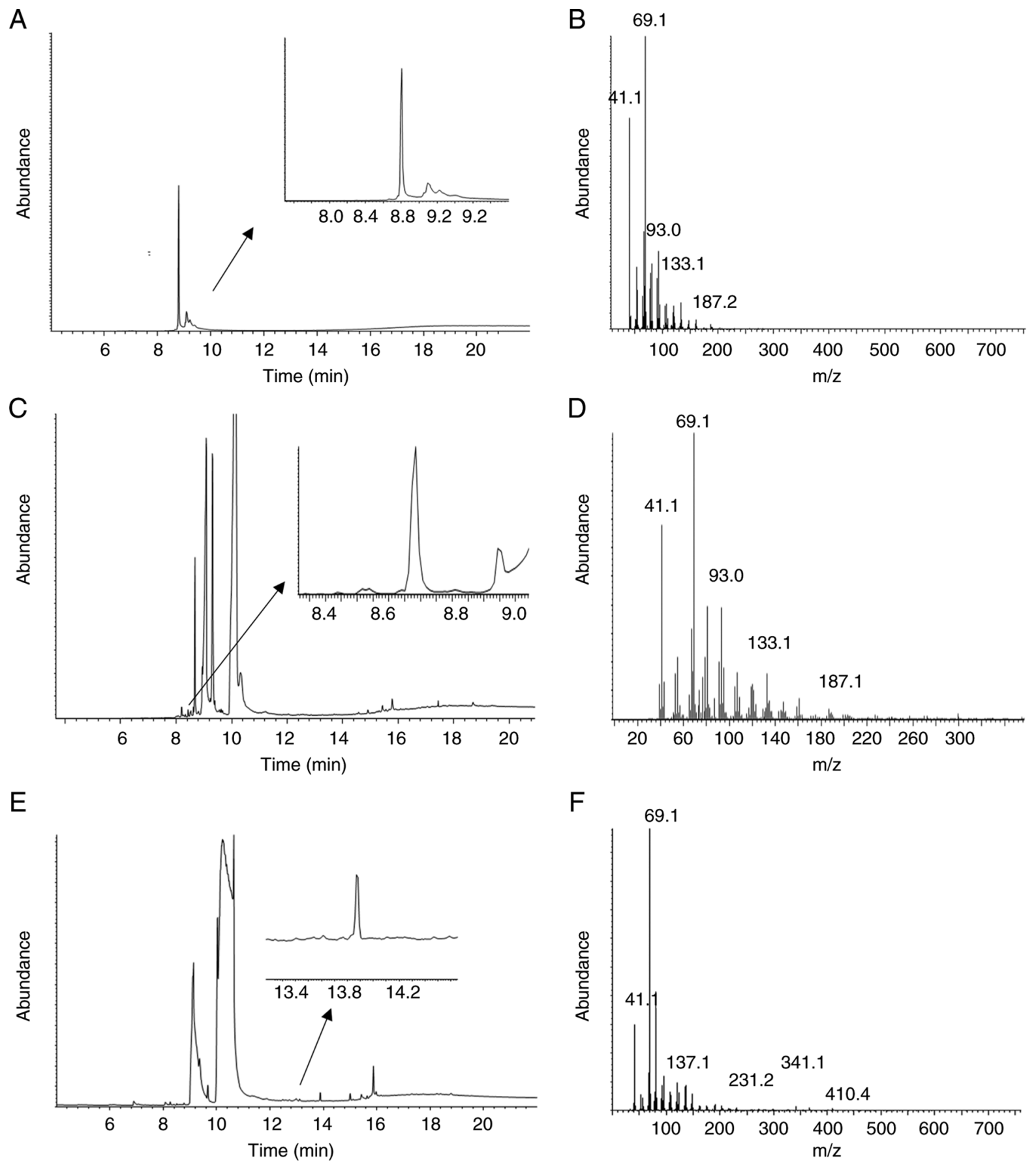

Figure 5. GC-MS analysis of geranylgeraniol derivatives in extract. (A) GC-MS chromatogram of geranylgeraniol and (B) the electron ionization mass spectrum of geranylgeraniol at $\mathrm{R}_{\mathrm{t}} 8.82 \mathrm{~min}$. (C) GC-MS chromatogram of lettuce extract and (D) the electron ionization mass spectrum of a geranylgeraniol derivative at $\mathrm{R}_{\mathrm{t}} 8.69 \mathrm{~min}$. (E) GC-MS chromatogram of bean extract and $(\mathrm{F})$ the electron ionization mass spectrum of a geranylgeraniol derivative at $\mathrm{R}_{\mathrm{t}} 13.88 \mathrm{~min}$. $\mathrm{R}_{\mathrm{t}}$, retention time.

The extract from lettuce substantially suppressed the activity of pitavastatin, however the extract on its own stimulated cell growth by up to $40 \%$. Most of the extracts from foods rich in carbohydrate (Fig. 2C) had no activity but an extract from oats was also able to rescue the effects of pitavastatin. Finally, we tested various other foods stuffs (Fig. 2D). Among these, extracts from pecan nuts and boiled eggs suppressed the effects of pitavastatin, although these extracts were toxic on their own when tested at high concentrations and in the absence of pitavastatin, precluding full exploration of their activity. We also noted that a commercially available pasta sauce suppressed the activity of pitavastatin, possibly reflecting its high sunflower oil content. To confirm these results were not unique to Ovcar-4 cells, we again used Fuov-1 cells and tested two extracts that were able to suppress the activity of pitavastatin in Ovcar-4 cells (sunflower oil, lettuce) and two extracts which did not (fig, bread). A similar pattern of activity was seen in the Fuov-1 cells to that observed in the Ovcar-4 cells (Fig. 3).

We have previously shown that pitavastatin induces cell death through apoptosis (8). To confirm here that the effects in the cell growth assay reflected cell death, we measured cell 
viability after exposure to pitavastatin using two extracts, one which significantly rescued the activity of pitavastatin in the cell growth assay (sunflower oil) and one which did not (milk). Consistent with this, exposure to pitavastatin resulted in cell death when assessed by trypan blue staining and this was suppressed by addition of sunflower oil extract, but not by an extract from milk (Fig. 4).

We next used GC-MS to confirm the presence of geranylgeraniol in two of the extracts that rescued the effect of pitavastatin (Fig. 5). Analysis of the lettuce extract identified free geranylgeraniol (Fig. 5C and D). Free geranylgeraniol was not detected in the bean extract by GCMS (Fig. 5E and F), however a derivative which contained geranylgeraniol (Fig. 5A) was identified.

\section{Discussion}

We have previously shown that the potential activity of statins as a cancer treatment depends on the reduced production of geranylgeraniol. However, several human foods have been reported to contain geranylgeraniol, potentially bypassing the effect of the statins on the cancer cells. Here we have attempted to enable clinical trials of statins by beginning to delineate which foods do not interfere with the cytotoxic activity of statins and so may be eaten by patients while using statins to treat cancer.

We have previously shown that geranylgeraniol, but not farnesol, reverses the activity of pitavastatin or simvastatin $(6,8)$. Here we extended this and tested other products of the mevalonate pathway whose biosynthesis would also be anticipated to be blocked by statins. However, addition of coenzyme Q, dolichol or cholesterol had no effect on the activity of pitavastatin whereas geranylgeraniol did. We therefore used a method to prepare extracts from a range of foods (oils, meals, fruits and vegetables) previously used to extract geranylgeraniol. However, we used a bioassay to evaluate the activity of these extracts, rather than quantifying geranylgeraniol by analytical methods. This approach has the advantage that it makes no assumption about the nature and quantities of the compounds present which can interfere with the activity of the statin. There may be other molecules other than geranylgeraniol which have yet to be identified which suppress the activity of statins. Consistent with our previous results, the sunflower extract reversed the activity of pitavastatin to an extent comparable to that achieved with geranylgeraniol itself, as did corn oil. On the other hand, some extracts such as groundnut oil had more modest effects while others such as rape seed oil showed no significant effect on the anti-cancer activity of pitavastatin. We also found that foods other than oils, particularly beans, cherries, eggs and oats, suppressed the activity of pitavastatin but extracts from several other carbohydrates and fruits had no effect. The extract from lettuce also rescued the effect of pitavastatin and GC-MS analysis confirmed the presence of geranylgeraniol. However, the extract also stimulated cell growth on its own, confusing interpretation. It is possible that the extract contains an additional growth stimulatory molecule that remains to be identified. Taken together, our data suggest some foods are more likely than others to interfere with the anti-cancer activity of statins.
To confirm that the observations were not unique to Ovcar- 4 cells, we also tested the ability of mevalonate pathway metabolites and selected food extracts to suppress the activity of pitavastatin in Fuov-1 cells. Comparable results were obtained, although the lettuce extract suppressed the activity of pitavastatin while having no growth promoting properties in Fuov-1 cells. We have also previously shown that extracts from sunflower and olive oil and rice suppress the cytotoxic activity of pitavastatin in Ovcar-3 and Ovcar-8 cells (6). In all of these cell lines, geranylgeraniol suppressed the activity of pitavastatin. This suggests that the ability of various extracts to suppress the cytotoxic activity of pitavastatin are not unique to Ovcar-4 cells but are likely to apply to ovarian cancer cells in general and possibility cancer cells from other tissues.

In this study, we measured the effect of the extracts on the cytotoxicity of pitavastatin primarily by staining the cells with sulforhodamine B. This assay measures total cellular protein, which is often used as a surrogate for cell number and consequently reflects the growth of a cell culture rather than directly measuring cell death. We confirmed that the pitavastatin caused cell death and that this could be rescued by certain extracts by using the trypan blue assay. This method has the advantage that it measures cell death independently of the mode of cell death. However, we have previously shown that pitavastatin induces apoptosis in Ovcar-3, Ovcar-8, Ovsaho, Cov362, SkOv-3, Igrov-1 and A2780 ovarian cancer cells. This was assessed by several methods including activation of both executioner caspases-3/7 and initiator caspases (caspase-8, caspase-9), PARP cleavage and annexin/PI staining $(4,6,10)$. In the case of Ovcar-3 and Ovcar-8, we also showed that geranylgeraniol and extracts from sunflower oil, olive oil and rice suppress caspase-3/7 activation by pitavastatin (6). These data suggest food extracts can suppress statin-induced cell death. However, the potential clinical use of statins in oncology may extend beyond inducing tumour cell apoptosis. Others have also shown that statins can inhibit other cancer hallmarks, including migration, invasion and cell progression $(2,3)$ and further studies to evaluate the activity of the food extracts on these hallmarks are desirable.

Previous research has used analytical methods such as GC-MS to identify either free geranylgeraniol or its derivatives in several human foodstuffs. Geranylgeraniol has been discovered as a wax ester in several oils, in particular oils prepared from sunflower $(7,11)$ vegetable $(12)$, linseed $(13)$, soybean (7), sesame (7), hemp (14) and olives (15). The potent effect of the sunflower oil extract in suppressing the cytotoxic effect of pitavastatin we observed is particularly significant because apart from its use to fry food, sunflower oil is also an ingredient found in approximately 1,000 manufactured food products (16). Oil extracted from hazelnuts, pecans and almonds also have been reported to contain geranylgeraniol $(17,18)$. In contrast to these oils, rapeseed oil lacks at least the common 22 and 24 carbon fatty acid esters of geranylgeraniol (7) and extracts from rapeseed oil also failed to inhibit the activity of pitavastatin in our experiments. This suggests that this oil may be preferable for patients to use for culinary purposes while they are receiving statins to treat cancer. Geranylgeraniol is also found in certain types of rice (9) and we have previously shown that a rice extract suppresses the cytotoxic effect of pitavastatin (6). Leaves 
from E. persicus, a traditional food in central Asia and the Middle East (19) and fruit from Pterodon tree, which his used in ethnomedicine in Brazil (20) have also been reported to contain geranylgeraniol. Our own GC-MS analysis identified free geranylgeraniol in the extract from lettuce. However, it is possible that the geranylgeraniol is present in another form, such as an ester, in lettuce itself and that it is liberated by the alkaline hydrolysis step in the extraction method. The extract obtained from beans contained a derivative of geranylgeraniol, rather than free geranylgeraniol. Further investigation is necessary to confirm its precise chemical identity; it may be a wax ester that is not susceptible to alkaline hydrolysis but geranylgeraniol is liberated enzymatically from it in the cancer cells. Lastly, we note that phytol, derived from the reduction of geranylgeraniol, is a component of chlorophyll (21). This suggests that at least trace quantities of geranylgeraniol are likely to occur throughout the plant kingdom.

Based on these observations, we suggest that patients may inadvertently consume sufficient geranylgeraniol to counter the anti-cancer activity of statins. Consequently, a diet certified to lack geranylgeraniol may be beneficial for patients using statins to treat cancer. This would preferably be achieved by establishing a database of foods in which geranylgeraniol and its derivatives have been systematically quantified by appropriate analytical methods. Until this is available, the data presented here may be of help in identifying such a diet. In particular, we suggest patients using statins to control cancer should consider avoiding sunflower oil or corn oil, nuts, eggs, oats, beans, lettuce and cherries. The foods which failed to suppress the activity of pitavastatin on cells are likely to be improved choices for patients using statins to treat cancer and which may be consumed alongside food replacements such as Ensure which we have shown also do not interfere with the activity of statins (6). It may also be preferable to avoid pre-prepared food products that are rich in oils or ingredients that have not been evaluated. For example, a commercial pasta sauce we tested suppressed the cytotoxic activity of pitavastatin.

Several issues remain to be addressed. We anticipate that the food extracts are able to restore membrane localization of key signalling proteins such as small GTPases by restoring their geranylgeranylation. We have previously shown that pitavastatin decreases the proportion of rho, ras, cdc42 and rab6A in cell membranes (4). Furthermore, statins reduce the amount of Rab7, presumably as a result of turnover following reduced membrane localization, and that this is reversed by addition of geranylgeraniol (8). However, we have not yet formally shown that the food extracts are able to restore geranylgeranylation and membrane localization of small GTPases. In addition, in most cases we have neither fully identified nor quantified the compounds in the foods which suppress the activity of pitavastatin. Although they are likely to be geranylgeraniol derivatives, it is possible other compounds can inhibit the activity of pitavastatin and that remain to be identified. We do not know the bioavailability of the various geranylgeraniol derivatives, nor do we know the amount of geranylgeraniol that must be absorbed to suppress the cytotoxic activity of statins in patients. Thus, we acknowledge that it remains a formal possibility that the ingestion of foods containing geranylgeraniol has minimal effect on the activity of statins as anti-cancer agents because insufficient geranylgeraniol reaches the systemic circulation from dietary sources. Further research is essential to address this. Until then, we consider that when clinical trials of statins in cancer are conducted, it is prudent to minimize dietary geranylgeraniol to maximize the chances of the trials being successful. An indication of anti-cancer activity of a statin in a prospective clinical trial would provide significant motivation to carry out a more thorough analysis of geranylgeraniol in human food. We have only so far tested a limited number of foods and a wider range would facilitate compliance with a 'geranylgeraniol-free' diet. This work also raises the question whether other targeted cancer therapeutics could be affected by diet. This overlooked area warrants additional research.

\section{Acknowledgements}

Not applicable.

\section{Funding}

The present study was funded by the Iraqi Ministry of Higher Education and Scientific research (MOHESR; grant nos. S1884 and S939).

\section{Availability of data and materials}

The datasets used and/or analysed during the current study are available from the corresponding author on reasonable request.

\section{Authors' contributions}

The project was conceived by AR. The extracts were prepared and tested by MJJ, SI, MK and CB. GCMS was performed by MJJ and WL. AR, SI, MK and CB analysed the biological data. WW and MJJ analysed the GCMS data. AR, MJJ and WW confirm the authenticity of all the raw data. All authors have read and approved the final manuscript.

\section{Ethics approval and consent to participate}

Not applicable.

\section{Patient consent for publication}

Not applicable.

\section{Competing interests}

The authors declare that they have no competing interests.

\section{References}

1. Rooth C: Ovarian cancer: Risk factors, treatment and management. Br J Nurs 22: S23-S30, 2013.

2. Mullen PJ, Yu R, Longo J, Archer MC and Penn LZ: The interplay between cell signalling and the mevalonate pathway in cancer. Nat Rev Cancer 16: 718-731, 2016.

3. Altwairgi AK: Statins are potential anticancerous agents (Review). Oncol Rep 33: 1019-1039, 2015. 
4. Abdullah MI, Abed MN and Richardson A: Inhibition of the mevalonate pathway augments the activity of pitavastatin against ovarian cancer cells. Sci Rep 7: 8090, 2017.

5. Abdullah MI, de Wolf E, Jawad MJ and Richardson A: The poor design of clinical trials of statins in oncology may explain their failure-lessons for drug repurposing. Cancer Treat Rev 69: 84-89, 2018.

6. de Wolf E, Abdullah MI, Jones SM, Menezes K, Moss DM, Drijfhout FP, Hart SR, Hoskins C, Stronach EA and Richardson A: Dietary geranylgeraniol can limit the activity of pitavastatin as a potential treatment for drug-resistant ovarian cancer. Sci Rep 7: 5410, 2017.

7. Biedermann M, Haase-Aschoff $\mathrm{P}$ and Grob K: Wax ester fraction of edible oils: Analysis by on-line LC-GC-MS and GCxGC-FID. Eur J Lipid Sci Technol 110: 1084-1094, 2008.

8. Robinson E, Nandi M, Wilkinson LL, Arrowsmith DM, Curtis AD and Richardson A: Preclinical evaluation of statins as a treatment for ovarian cancer. Gynecol Oncol 129: 417-424, 2013.

9. Muraguchi T, Okamoto K, Mitake M, Ogawa H and Shidoji Y: Polished rice as natural sources of cancer-preventing geranylgeranoic acid. J Clin Biochem Nutr 49: 8-15, 2011.

10. Abdullah MI, Abed MN, Khanim F and Richardson A: Screening a library of approved drugs reveals that prednisolone synergizes with pitavastatin to induce ovarian cancer cell death. Sci Rep 9 9632, 2019.

11. Reiter B and Lorbeer E: Analysis of the wax ester fraction of olive oil and sunflower oil by gas chromatography and gas chromatography-mass spectrometry. J Am Oil Chem Soc 78: 881-888, 2001.

12. Fedeli E and Jacini G: Lipid composition of vegetable oils. Adv Lipid Res 9: 335-382, 1971.
13. Fedeli E, Capella P, Cirimele M and Jacini G: Isolation of geranyl geraniol from the unsaponifiable fraction of linseed oil. J Lipid Res 7: 437-441, 1966.

14. Montserrat-de la Paz S, Marin-Aguilar F, Garcia-Gimenez MD and Fernandez-Arche MA: Hemp (Cannabis sativa L.) seed oil: Analytical and phytochemical characterization of the unsaponifiable fraction. J Agric Food Chem 62: 1105-1110, 2014.

15. Ranalli A, Modesti G, Patumi M and Fontanazza G: The compositional quality and sensory properties of virgin olive oil from a new olive cultivar-I-77. Food Chem 69: 37-46, 2000.

16. Gigandet S: Open food facts, 2021. https://world.openfoodfacts. org/cgi/search.pl?search_terms=sunflower+oil\&search_simple $=1 \&$ action=process. Acessed September 16, 2021 .

17. Fernandes GD, Gómez-Coca RB, Pérez-Camino MC, Moreda W and Barrera-Arellano D: Chemical characterization of major and minor compounds of nut oils: Almond, hazelnut, and pecan nut. J Chem 2017: $2609549,2017$.

18. Purcaro G, Barp L and Conte L: Comparison of different injection modes in edible oil minor components analysis. J Sep Sci 38: 2278-2285, 2015

19. Salehi B, Ayatollahi SA, Segura-Carretero A, Kobarfard F, Contreras MDM, Faizi M, Sharifi-Rad M, Tabatabai SA and Sharifi-Rad J: Bioactive chemical compounds in Eremurus persicus (joub. \& spach) Boiss. essential oil and their health implications. Cell Mol Biol (Noisy-Le-Grand) 63: 1-7, 2017.

20. Menna-Barreto R, Laranja GAT, Silva MCC, Coelho MGP, Paes MC, Oliveira MM and de Castro SL: Anti-trypanosoma cruzi activity of pterodon pubescens seed oil: Geranylgeraniol as the major bioactive component. Parasitol Res 103: 111-117, 2008.

21. Gutbrod K, Romer J and Dormann P: Phytol metabolism in plants. Prog Lipid Res 74: 1-17, 2019. 